\title{
CLASSICAL BI-POISSON PROCESS: AN INVERTIBLE QUADRATIC HARNESS
}

\author{
WŁODZIMIERZ BRYC AND JACEK WESOŁOWSKI
}

\begin{abstract}
We give an elementary construction of a time-invertible Markov process which is discrete except at one instance. The process is one of the quadratic harnesses studied in [5, 2], and 3. It can be regarded as a random joint of two independent Poisson processes.
\end{abstract}

\section{INTRODUCTION}

According to [11, a stochastic process $\left(X_{t}\right)_{t>0}$ has the time inversion property, if it has the same finite-dimensional distributions as the process $\left(t X_{1 / t}\right)_{t>0}$. In papers [6] and 8 the authors give criteria for the time-invertibility of Markov processes with transition probabilities that have smooth densities with respect to the Lebesgue measure.

In this note we give a new elementary example of a time-invertible Markov process for which all transitions except to time $t=1$ are discrete, see Proposition 4.2 This improves upon [4, Corollary 3.4], where we gave a less elementary example of a time-invertible Markov process that had transition probabilities with a discrete component. Both examples are particular cases of a more general family of Markov processes which in 22 we called the bi-Poisson processes. According to 2, Example 4.8 and Proposition 4.13], a bi-Poisson process with parameters $(\eta, \theta, q)$ is a squareintegrable Markov process $\left(X_{t}\right)$ which is uniquely determined by the following three properties:

$$
\begin{gathered}
\mathbb{E}\left(X_{t}\right)=0, \mathbb{E}\left(X_{t} X_{s}\right)=\min \{t, s\}, \\
\mathbb{E}\left(X_{t} \mid \mathcal{F}_{s, u}\right)=\frac{u-t}{u-s} X_{s}+\frac{t-s}{u-s} X_{u},
\end{gathered}
$$

(3) $\operatorname{Var}\left(X_{t} \mid \mathcal{F}_{s, u}\right)=$

$\frac{(u-t)(t-s)}{u-q s}\left(1+\eta \frac{u X_{s}-s X_{u}}{u-s}+\theta \frac{X_{u}-X_{s}}{u-s}-(1-q) \frac{\left(u X_{s}-s X_{u}\right)\left(X_{u}-X_{s}\right)}{(u-s)^{2}}\right)$,

for all $0 \leq s<t<u$, where

$$
\mathcal{F}_{s, u}=\sigma\left\{X_{t}: 0 \leq t \leq s \text { or } t>u\right\} .
$$

Date: August 18, 2005.

Printed 6:00 am, November 20, 2018 File: bi-1-Poisson8.TEX.

2000 Mathematics Subject Classification. 60J25.

Research partially supported by NSF grants \#INT-0332062, \#DMS-0504198, and by the C.P. Taft Memorial Fund. 
Property (2) says that the bi-Poisson process is a harness, see [9]. Condition (3) means that it is a quadratic harnesses, see 2]. The adjective "classical" refers to the value of parameter $q=1$, compare [2, Section 4.2] and [5. Section 4.2]. In Proposition 4.1 we show that $\left(X_{t}\right)$ can be constructed by joining together two independent Poisson processes with the same random gamma intensity. This is accomplished by appropriate affine transformations and deterministic changes of time.

In 4] we use orthogonal polynomials to construct the transition probabilities of the bi-Poisson process when $q=0$, and we show that its univariate distributions form a semigroup with respect to a certain generalized convolution related to free probability. The univariate distributions of the general bi-Poisson process were implicitly identified in [2, Example 4.8], and the corresponding Markov process is under construction in [3]. This construction relies heavily on cumbersome identities between certain multi-parameter families of orthogonal polynomials, and identifies the transition probabilities in implicit form only. However, when $q=1$, the explicit transitions probabilities can be read out. They turn out to be related to the pure birth and the pure death processes and are amenable to explicit elementary analysis.

In this paper we present an elementary construction of the bi-Poisson process with parameters $(\eta, \theta, 1)$. Throughout most of the paper the value of the third parameter is fixed as $q=1$, in which case we say that we consider a bi-Poisson process with parameters $(\eta, \theta)$, skipping the third parameter of the triple.

The plan of the paper is as follows. In Section 2 we give the construction of the process. In Section 3 we verify that the construction indeed gives a bi-Poisson process. In Section 4 we deduce some additional properties, including time-invertibility.

\section{Construction}

It is known, see [2] Proposition 4.13] that a bi-Poisson process $\left(X_{t}\right)$ with parameters $(\eta, \theta)$ satisfies $\eta \theta \geq 0$.

In the degenerate case $\theta \eta=0$ it is known that the bi-Poisson process $\left(X_{t}\right)$ is either $X_{t}=B_{t}$, where $\left(B_{t}\right)$ is the standard Brownian motion for $\eta=\theta=0$, or $X_{t}=\theta N_{t / \theta^{2}}-t / \theta$, where $\left(N_{t}\right)$ is the standard Poisson process when $\eta=0$, $\theta \neq 0$, see [12, Theorem 1]. Passing to the time inverse $\left(t X_{1 / t}\right)$, we see that $X_{t}=\eta t N_{1 /\left(t \eta^{2}\right)}-1 / \eta$ in the remaining degenerate case $\theta=0, \eta \neq 0$.

We will therefore concentrate on the case $\theta \eta>0$. Passing to $\left(-X_{t}\right)$ preserves (3) replacing parameters $(\eta, \theta)$ by $(-\eta,-\theta)$, so we may assume $\eta, \theta>0$. Replacing process $\left(X_{t}\right)$ by process $\left(\sqrt{\eta / \theta} X_{t \theta / \eta}\right)$, we get the bi-Poisson process with parameters $(\sqrt{\eta \theta}, \sqrt{\eta \theta})$. Thus without loss of generality we may assume that $\eta=\theta>0$.

The moment of time $t=1$ is preserved by the time-inversion and plays a special role in the construction. The bi-Poisson process traverses a family of deterministic lines, with jumps in the upwards direction when $t<1$ and in the downwards direction when $t>1$, see Fig. 1.

The process is determined by specifying an integer that describes the line being followed at time $t$. The integers that describe the upwards jumps form a linear pure birth process with immigration with the time transformed to run on the interval $[0,1)$. At time $t=1$ instead of being infinite, the process takes the continuous spectrum of real values. For $t>1$, the downwards jumps form a linear pure death process which "returns from $\infty$ " by a Poisson entrance law, again with the time transformed to run on the interval $(1, \infty)$. The deterministic time transformations 


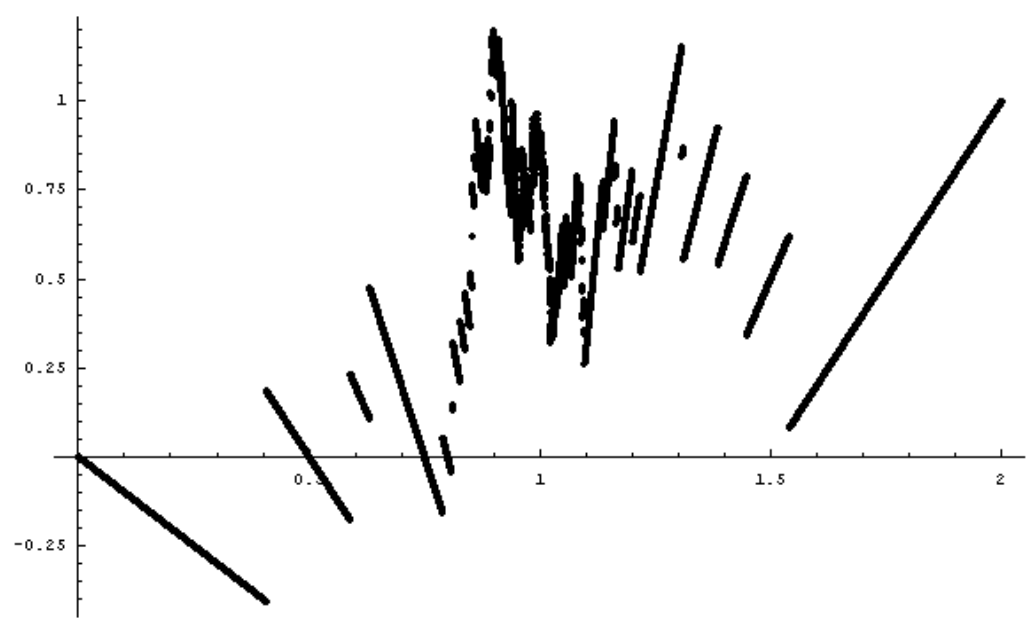

Figure 1. Simulated sample trajectory of the bi-Poisson process. The process follows the segments $\ell_{j}: y=\theta(1-t) j-t / \theta, 0<t<1$, $j=0,1,2, \ldots$ except for the upwards jumps, and then follows the half-lines $y=\theta(t-1) j-1 / \theta, t>1, j=\ldots, 1,0$ with the downwards jumps.

are logarithmic and introduce a rather simple non-homogeneity into the birth rates and the death rates of the process. However, they force infinite number of jumps before and after $t=1$.

For a more formal description of $\left(X_{t}\right)$, we set

$$
X_{t}= \begin{cases}\theta(1-t) Z_{t}-\frac{t}{\theta}, & 0 \leq t<1 \\ \theta Z_{1}-\frac{1}{\theta}, & t=1 \\ \theta(t-1) Z_{t}-\frac{1}{\theta}, & t>1\end{cases}
$$

where random variables $Z_{t}$ are $\{0,1,2, \ldots\}$-valued for $t \neq 1$. We will construct the appropriate process $\left(Z_{t}\right)_{t \geq 0}$ in three steps: we first define $\left(Z_{t}\right)_{0 \leq t<1}$ as a pure birth process, then we extend it to $t=1$ by passing to the limit, and finally we extend the process to $t>1$ as a pure death process with a $Z_{1}$-dependent Poisson entrance law.

2.1. The pure birth phase. As $\left(Z_{t}\right)_{0 \leq t<1}$ we take the non-homogeneous linear pure birth process with immigration with the birth rate

$$
\lambda_{n}(t)=\frac{n+\frac{1}{\theta^{2}}}{1-t}
$$

The properties of such a process are well known. For small enough $|z|$, 10, Exercise 5.1] gives the following generating function of the transition probabilities for the more general non-homogeneous linear pure birth process with the birth rate $\lambda_{n}(t)=$ $\nu(t)+n \lambda(t)$.

$$
\sum_{k=0}^{\infty} z^{k} p_{j, j+k}(s, t)=z^{\nu(s) / \lambda(s)-\nu(t) / \lambda(t)}\left(\frac{p(s, t)}{1-z(1-p(s, t))}\right)^{j+\nu(s) / \lambda(s)}
$$


where $p(s, t)=e^{-\int_{s}^{t} \lambda(u) d u}$. In our setting, $p(s, t)=\frac{1-t}{1-s}$ and with $z=e^{u(1-t)}$ we get

$$
\mathbb{E}\left(e^{u(1-t)\left(Z_{t}-Z_{s}\right)} \mid \mathcal{F}_{\leq s}\right)=\left(\frac{1-t}{1-s-(t-s) e^{u(1-t)}}\right)^{Z_{s}+1 / \theta^{2}},
$$

where $\mathcal{F}_{\leq s}=\sigma\left\{X_{r}: r \leq s\right\}=\sigma\left\{Z_{r}: r \leq s\right\}$. Thus the conditional distribution $\mathcal{L}\left(Z_{t}-Z_{s} \mid Z_{s}\right)$ is negative binomial with parameters $r=Z_{s}+1 / \theta^{2}, p=(1-t) /(1-s)$. (Table 1 lists the parameterizations of the distributions we use in this note.)

\begin{tabular}{||c|c|c|c||}
\hline \hline Name & Parameters & Distribution & $E\left(e^{u Z}\right)$ \\
\hline \hline Poisson & $\lambda>0$ & $e^{-\lambda} \lambda^{k} / k !, k=0,1,2, \ldots$ & $\exp \left(\lambda\left(e^{u}-1\right)\right)$ \\
Gamma & $p>0, \sigma>0$ & $f(z)=\frac{1}{\sigma^{p} \Gamma(p)} x^{p-1} e^{-x / \sigma}$ & $(1-\sigma u)^{-p}$ \\
Negative Binomial & $r>0,0<p<1$ & $\frac{\Gamma(k+r)}{\Gamma(r) k !} p^{r}(1-p)^{k}, k=0,1, \ldots$ & $\frac{p^{r}}{\left(1-(1-p) e^{u}\right)^{r}}$ \\
Binomial & $n \geq 0,0 \leq p \leq 1$ & $\left(\begin{array}{l}n \\
k\end{array}\right) p^{k}(1-p)^{n-k}, k=0,1, \ldots, n$. & $\left(1-p+p e^{u}\right)^{n}$ \\
\hline \hline
\end{tabular}

TABLE 1. Laws that appear as transition probabilities.

Differentiating (5) at $u=0$ and using (4) we verify that $\left(X_{t}, \mathcal{F}_{\leq t}\right)_{0 \leq t<1}$ is a martingale. Setting $s=0$, from (5) we get $\mathbb{E}\left(X_{t}\right)=0$ and differentiating (5) again, after a calculation we get $\mathbb{E}\left(X_{t}^{2}\right)=t$.

In particular, $X_{1}=\lim _{t \rightarrow 1} X_{t}$ converges almost surely, as does $Z_{1}=\lim _{t \rightarrow 1}(1-$ t) $Z_{t}$. Taking the limit in (5) we see that

$$
\mathbb{E}\left(e^{u Z_{1}} \mid Z_{s}\right)=(1-u(1-s))^{-Z_{s}-1 / \theta^{2}},
$$

thus $\mathcal{L}\left(Z_{1} \mid Z_{s}\right)$ is gamma with shape parameter $p=Z_{s}+\frac{1}{\theta^{2}}$ and scale parameter $\sigma=1-s$, see Table 1. In particular, $Z_{1}$ is gamma with $r=1 / \theta^{2}, \sigma=1$, and the support of $X_{1}$ is $[-1 / \theta, \infty)$.

2.2. The pure death phase. We now extend $\left(Z_{t}\right)$ from $0 \leq t \leq 1$ to $t>1$ by specifying $\left(Z_{t}\right)_{t>1}$ as a pure death process with the death rate

$$
\mu_{n}(t)=\frac{n}{t-1},
$$

and the $Z_{1}$-dependent entrance law $\mathcal{L}\left(Z_{t} \mid Z_{1}\right)$ which we take as the Poisson law with parameter $\lambda=Z_{1} /(t-1)$. Thus

$$
\mathbb{E}\left(e^{u Z_{t}} \mid Z_{1}\right)=\exp \left(Z_{1} \frac{e^{u}-1}{t-1}\right)
$$

A well known property of the linear pure birth process is that for $1<s<t$ the transition probabilities $\mathcal{L}\left(Z_{t} \mid Z_{s}\right)$ are binomial with parameters $n=Z_{s}, p=$ $(s-1) /(t-1)$, so

$$
\mathbb{E}\left(e^{u Z_{t}} \mid Z_{s}\right)=\left(\frac{t-s+(s-1) e^{u}}{t-1}\right)^{Z_{s}} .
$$

The Poisson distribution is indeed the entrance law: given $1<s<t$ we have

$$
\operatorname{Pr}\left(Z_{t}=i \mid Z_{1}\right)=\sum_{n=i}^{\infty} \operatorname{Pr}\left(Z_{t}=i \mid Z_{s}=n\right) \operatorname{Pr}\left(Z_{s}=n \mid Z_{1}\right) .
$$


Indeed, the right hand side is

$$
\frac{Z_{1}^{i}}{i !(t-1)^{i}} e^{-Z_{1} /(s-1)} \sum_{n=i}^{\infty} \frac{Z_{1}^{n-i}(t-s)^{n-i}}{(n-i) !(s-1)^{n-i}(t-1)^{n-i}}=\frac{Z_{1}^{i}}{i !(t-1)^{i}} e^{-Z_{1} /(t-1)} .
$$

We now verify that the two pieces of the process fit together into a well defined Markov process $\left(Z_{t}\right)_{t \geq 0}$. For $0 \leq s<1<t$, by conditioning on $Z_{1}$ we get

$$
\begin{gathered}
\operatorname{Pr}\left(Z_{t}=j \mid Z_{s}\right)=\frac{1}{j !(t-1)^{j}} \mathbb{E}\left(Z_{1}^{j} e^{-Z_{1} /(t-1)} \mid Z_{s}\right) \\
=\int_{0}^{\infty} \frac{x^{Z_{s}+j+1 / \theta^{2}-1}}{j !(t-1)^{j}(1-s)^{Z_{s}+1 / \theta^{2}} \Gamma\left(Z_{s}+1 / \theta^{2}\right)} e^{-x /(1-s)} d x \\
=\frac{\Gamma\left(i+j+1 / \theta^{2}\right)}{j ! \Gamma\left(i+1 / \theta^{2}\right)}\left(\frac{t-1}{t-s}\right)^{i+1 / \theta^{2}}\left(\frac{1-s}{t-s}\right)^{j} .
\end{gathered}
$$

Thus $\mathcal{L}\left(Z_{t} \mid Z_{s}\right)$ is negative binomial with $r=Z_{s}+1 / \theta^{2}$ and $p=(t-1) /(t-s)$. In particular, $Z_{t}$ is negative binomial with $r=1 / \theta^{2}$ and $p=1-1 / t$. An elementary calculation shows that $X_{t}$ defined by (4) has mean zero and variance $t$.

A straightforward calculation leads now to the verification of the ChapmanKolmogorov equations in the remaining two cases:

(i) If $0<s_{1}<s_{2}<1<t$ and $i, k \geq 0$ then

$$
\operatorname{Pr}\left(Z_{t}=k \mid Z_{s_{1}}=i\right)=\sum_{j=i}^{\infty} \operatorname{Pr}\left(Z_{t}=k \mid Z_{s_{2}}=j\right) \operatorname{Pr}\left(Z_{s_{2}}=j \mid Z_{s_{1}}=i\right) .
$$

Indeed, the right hand side is

$$
\begin{gathered}
\frac{1}{k ! \Gamma\left(i+1 / \theta^{2}\right)}\left(\frac{t-1}{1-s_{1}}\right)^{i+1 / \theta^{2}} \sum_{j=i}^{\infty} \frac{\Gamma\left(j+k+1 / \theta^{2}\right)\left(1-s_{2}\right)^{i+k+1 / \theta^{2}}(t-1)^{j-i}\left(s_{2}-s_{1}\right)^{j-i}}{(j-i) !\left(1-s_{1}\right)^{j-i}\left(t-s_{2}\right)^{j-i}\left(t-s_{2}\right)^{i+k+1 / \theta^{2}}} \\
=\frac{\Gamma\left(i+k+1 / \theta^{2}\right)}{k ! \Gamma\left(i+1 / \theta^{2}\right)}\left(\frac{t-1}{1-s_{1}}\right)^{i+1 / \theta^{2}} \frac{\left(1-s_{2}\right)^{i+k+1 / \theta^{2}}}{\left(t-s_{2}\right)^{i+k+1 / \theta^{2}}}\left(1-\frac{(t-1)\left(s_{2}-s_{1}\right)}{\left(1-s_{1}\right)\left(t-s_{2}\right)}\right)^{-\left(i+k+1 / \theta^{2}\right)} \\
=\frac{\Gamma\left(i+k+1 / \theta^{2}\right)}{k ! \Gamma\left(i+1 / \theta^{2}\right)}\left(\frac{t-1}{t-s_{1}}\right)^{i+1 / \theta^{2}}\left(\frac{1-s_{1}}{t-s_{1}}\right)^{k} .
\end{gathered}
$$

(ii) If $0<s<1<t_{1}<t_{2}$ and $i, k \geq 0$ then

$$
\operatorname{Pr}\left(Z_{t_{2}}=k \mid Z_{s}=i\right)=\sum_{n=k}^{\infty} \operatorname{Pr}\left(Z_{t_{t}}=k \mid Z_{t_{1}}=n\right) \operatorname{Pr}\left(Z_{t_{1}}=n \mid Z_{s}=i\right) .
$$

Indeed, the right hand side is

$$
\begin{gathered}
\frac{(1-s)^{k}\left(t_{1}-1\right)^{i+k+1 / \theta^{2}}}{k ! \Gamma\left(i+1 / \theta^{2}\right)\left(t_{1}-s\right)^{i+k+1 / \theta^{2}\left(t_{2}-1\right)^{k}}} \sum_{n=k}^{\infty} \frac{\Gamma\left(n+i+1 / \theta^{2}\right)}{(n-k) !}\left(\frac{\left(t_{2}-t_{1}\right)(1-s)}{\left(t_{2}-1\right)\left(t_{1}-s\right)}\right)^{n-k} \\
=\frac{\Gamma\left(k+i+1 / \theta^{2}\right)}{k ! \Gamma\left(i+1 / \theta^{2}\right)}\left(\frac{t_{1}-1}{t_{2}-s}\right)^{i+1 / \theta^{2}}\left(\frac{1-s}{t_{2}-s}\right)^{k} .
\end{gathered}
$$

Thus $\left(Z_{t}\right)_{t \geq 0}$ is a well defined Markov process which determines Markov process $\left(X_{t}\right)_{t \geq 0}$ through the one-to-one transformation (4). 


\section{Conditional moments}

We now verify that $\left(X_{t}\right)_{t \geq 0}$ is a quadratic harness.

Theorem 3.1. For $\theta>0$, let $\left(Z_{t}\right)$ be the Markov process defined in previous Section. Let $\left(X_{t}\right)$ be defined by (4). Then $\left(X_{t}\right)$ is the bi-Poisson process with parameters $(\theta, \theta)$, i.e. it has covariance (1), conditional moments (2), and (3) with $\eta=\theta$ and $q=1$.

Proof. In Section 2 we already verified that $\mathbb{E}\left(X_{t}\right)=0, \mathbb{E} X_{t}^{2}=t$. Since $\mathcal{L}\left(Z_{t} \mid Z_{s}\right)$ is binomial for $1 \leq s<t$, we have $\mathbb{E}\left(Z_{t} \mid Z_{s}\right)=\frac{s-1}{t-1} Z_{s}$. Combining this with the already established martingale property for $t<1$, we see that $\left(X_{t}, \mathcal{F}_{\leq t}\right)_{t \geq 0}$ is a martingale. From the martingale property we get (11).

To compute the conditional moments, we calculate explicitly the conditional distribution of $\mathcal{L}\left(Z_{t} \mid Z_{s}, Z_{u}\right)$. These are routine calculations, so we just state the final answers, and omit most of the calculations of the corresponding moments.

(i) If $0<s<t<u<1$ then $\mathcal{L}\left(Z_{t}-Z_{s} \mid Z_{s}, Z_{u}\right)$ is binomial with parameters $n=Z_{u}-Z_{s}$ and $p=\frac{(1-u)(t-s)}{(1-t)(u-s)}$. Therefore

$$
\begin{gathered}
\mathbb{E}\left(Z_{t} \mid \mathcal{F}_{s, u}\right)=Z_{s}+\frac{(1-u)(t-s)}{(1-t)(u-s)}\left(Z_{u}-Z_{s}\right) \\
=\frac{(u-t)(1-s)}{(1-t)(u-s)} Z_{s}+\frac{(1-u)(t-s)}{(1-t)(u-s)} Z_{u} .
\end{gathered}
$$

Using (4) we get

$$
\mathbb{E}\left(X_{t} \mid \mathcal{F}_{s, u}\right)=-t / \theta+\frac{u-t}{u-s}\left(X_{s}+s / \theta\right)+\frac{t-s}{u-s}\left(X_{u}+u / \theta\right),
$$

which gives (2). Similarly,

$$
\operatorname{Var}\left(Z_{t} \mid \mathcal{F}_{s, u}\right)=\frac{(1-u)(t-s)(u-t)(1-s)}{(1-t)^{2}(u-s)^{2}}\left(Z_{u}-Z_{s}\right)
$$

which gives

$$
\operatorname{Var}\left(X_{t} \mid \mathcal{F}_{s, u}\right)=\frac{(t-s)(u-t)}{(u-s)^{2}}\left(\theta(1-s)\left(X_{u}+u / \theta\right)-\theta(1-u)\left(X_{s}+s / \theta\right)\right) \text {. }
$$

A calculation gives (3).

(ii) If $0<s<t<1<u$ then $\mathcal{L}\left(Z_{t}-Z_{s} \mid Z_{s}, Z_{u}\right)$ is negative binomial with parameters $r=Z_{s}+Z_{u}+1 / \theta^{2}$ and $p=\frac{(1-t)(u-s)}{(1-s)(u-t)}$. Therefore

$$
\begin{gathered}
\mathbb{E}\left(Z_{t} \mid \mathcal{F}_{s, u}\right)=Z_{s}+r(1-p) / p \\
=\frac{(1-s)(u-t)}{(1-t)(u-s)} Z_{s}+\frac{(u-1)(t-s)}{(1-t)(u-s)} Z_{u}+\frac{(u-1)(t-s)}{\theta^{2}(1-t)(u-s)}
\end{gathered}
$$

which leads to (2) and

$$
\begin{gathered}
\operatorname{Var}\left(Z_{t} \mid \mathcal{F}_{s, u}\right)=\frac{r(1-p)}{p^{2}} \\
=\frac{(u-1)(1-s)(t-s)(u-t)}{(1-t)^{2}(u-s)^{2}}\left(Z_{s}+Z_{u}+1 / \theta^{2}\right),
\end{gathered}
$$

which after a calculation leads to (3). 
(iii) If $0<s<1<t<u$ then $\mathcal{L}\left(Z_{t}-Z_{u} \mid Z_{s}, Z_{u}\right)$ is negative binomial with parameters $r=Z_{s}+Z_{u}+1 / \theta^{2}$ and $p=\frac{(t-1)(u-s)}{(t-s)(u-1)}$. A calculation verifies (2) and (3).

(iv) If $1<s<t<u$ then $\mathcal{L}\left(Z_{t}-Z_{u} \mid Z_{s}, Z_{u}\right)$ is binomial with $n=Z_{s}-Z_{u}$ and $p=\frac{(s-1)(u-t)}{(t-1)(u-s)}$. A calculation verifies (2) and (3).

The conditional moments for the remaining choices of $s<t<u$ follow by continuity.

\section{Additional Properties}

Proposition 4.1 (Poisson representation). Let $\left(N_{t}^{\lambda}\right)$ and $\left(M_{t}^{\lambda}\right)$ be two independent Poisson processes with intensity $\lambda>0$. If $\left(X_{t}\right)$ is a bi-Poisson process with positive parameters $(\eta, \theta)$ then

$\mathcal{L}\left(\left(t\left(h(t) X_{\frac{\theta}{\eta h(t)}}+\frac{1}{\eta}\right)\right)_{t>0},\left(t\left(X_{\frac{\theta h(t)}{\eta}}+\frac{1}{\eta}\right)\right)_{t>0} \mid X_{\theta / \eta}=\lambda-\frac{1}{\eta}\right)=\mathcal{L}\left(\left(N_{t}^{\lambda}\right)_{t>0},\left(M_{t}^{\lambda}\right)_{t>0}\right)$, where

$$
h(t)=\frac{1+\theta t}{\theta t} .
$$

Proof. Without loss of generality we assume $\eta=\theta$. By (4) and the Markov property, it suffices to prove that

$$
\mathcal{L}\left(\left(Z_{1 / h(t)}\right)_{t>0} \mid Z_{1}=\lambda\right)=\mathcal{L}\left(\left(N_{t}^{\lambda}\right)_{t>0}\right)
$$

and

$$
\mathcal{L}\left(\left(Z_{h(t)}\right)_{t>0} \mid Z_{1}=\lambda\right)=\mathcal{L}\left(\left(M_{t}^{\lambda}\right)_{t>0}\right)
$$

Both equalities follow now from elementary calculations of finite dimensional distributions using the conditional distributions identified in Section 2

To prove (10), take $t_{n}<t_{n-1}<\ldots t_{1}$ so that $1<h\left(t_{1}\right)<h\left(t_{2}\right)<\ldots h\left(t_{n}\right)$. Then for $k_{1} \geq k_{2} \geq \cdots \geq k_{n}$ denoting $Y_{j}=Z_{h\left(t_{j}\right)}$ we have

$$
\begin{gathered}
\operatorname{Pr}\left(Y_{1}=k_{1}, Y_{2}=k_{2}, \ldots, Y_{n}=k_{n} \mid Z_{1}=\lambda\right) \\
=\operatorname{Pr}\left(Y_{n}=k_{n} \mid Y_{n-1}=k_{n-1}\right) \ldots \operatorname{Pr}\left(Y_{2}=k_{2} \mid Y_{1}=k_{1}\right) \operatorname{Pr}\left(Y_{1}=k_{1} \mid Z_{1}=\lambda\right) \\
=\frac{\lambda^{k_{1}} \exp \left(-\lambda t_{1}\right)}{k_{n} !\left(k_{n-1}-k_{n}\right) ! \ldots\left(k_{1}-k_{2}\right) !} t_{n}^{k_{n}}\left(t_{n-1}-t_{n}\right)^{k_{n-1}-k_{n}} \ldots\left(t_{1}-t_{2}\right)^{k_{1}-k_{2}},
\end{gathered}
$$

which proves (10).

The proof of (9) is similar after using the generalized Bayes formula: for $t_{1}<t_{2}<$ $\ldots t_{n}$ so that $0<1 / h\left(t_{1}\right)<1 / h\left(t_{2}\right)<\ldots 1 / h\left(t_{n}\right)<1$. Then for $k_{1} \leq k_{2} \leq \cdots \leq k_{n}$, denoting $Y_{j}=Z_{1 / h\left(t_{j}\right)}$ we have

$$
\begin{gathered}
\operatorname{Pr}\left(Y_{1}=k_{1}, Y_{2}=k_{2}, \ldots, Y_{n}=k_{n} \mid Z_{1}=\lambda\right) \\
=\frac{f_{Z_{1} \mid Y_{n}=k_{n}}(\lambda)}{f_{Z_{1}}(\lambda)} \operatorname{Pr}\left(Y_{n}=k_{n} \mid Y_{n-1}=k_{n-1}\right) \ldots \operatorname{Pr}\left(Y_{2}=k_{2} \mid Y_{1}=k_{1}\right) \operatorname{Pr}\left(Y_{1}=k_{1}\right),
\end{gathered}
$$

where $f_{Z_{1} \mid Y_{n}=k_{n}}$ is the conditional density of $Z_{1}$ given $Y_{n}=k_{n}$, and $f_{Z_{1}}$ is the density of $Z_{1}$, which are both gamma, see the last paragraph of Section[2.1. Elementary calculations now prove (9).

Proposition 4.2 (Time-inversion). If $\left(X_{t}\right)$ is a bi-Poisson process with parameters $(\theta, \theta, q)$, then $\left(t X_{1 / t}\right)_{t>0}$ has the same distribution as $\left(X_{t}\right)_{t>0}$ (Compare [6], [8].) 
Proof. This follows from the fact that $\left(t X_{1 / t}\right)$ satisfies (11), (2), and (3), and hence by [2] Theorem 4.5 and Proposition 4.13] is determined uniquely. For $q=1$, the conclusion can also be derived directly from (4) and the fact that Markov process $\left(Z_{t}\right)_{t>0}$ has the same transition probabilities as $\left(Z_{1 / t}\right)_{t>0}$.

Proposition 4.3 (Distribution of upward jumps). For a bi-Poisson process $\left(X_{t}\right)$ with parameters $(\theta, \theta)$, where $\theta>0$, define

$$
\Gamma_{i}=\sup \left\{s \in[0,1): Z_{s}=i\right\}, \quad i=0,1, \ldots,
$$

i.e. $\Gamma_{i}$ is the time of the $(i+1)$-th jump of the process $\left(X_{t}\right)$ from the line $y=$ $\theta(1-t) i-t / \theta, 0 \leq t<1$, for $i=0,1, \ldots$, see Fig. [1. Then the joint density of the random vector $\left(\Gamma_{0}, \Gamma_{1}, \ldots, \Gamma_{k}\right)$ is

$$
f_{\left(\Gamma_{0}, \Gamma_{1}, \ldots, \Gamma_{k}\right)}\left(s_{0}, s_{1}, \ldots, s_{k}\right)=\frac{\Gamma\left(\frac{1}{\theta^{2}}+k+1\right)\left(1-s_{k}\right)^{\frac{1}{\theta^{2}}+k-1}}{\Gamma\left(\frac{1}{\theta^{2}}\right)\left(1-s_{0}\right)^{2}\left(1-s_{1}\right)^{2} \ldots\left(1-s_{k-1}\right)^{2}}
$$

for $0 \leq s_{0}<s_{1}<\ldots<s_{k}<1$ (and 0 otherwise).

Proof. Let $\left(M_{t}\right)_{t>0}$ be a homogeneous pure birth process with birth rates $\lambda_{n}=$ $n+1 / \theta^{2}, n=0,1, \ldots$ It is well known that the sojourn times $\tau_{j}$ of $\left(M_{t}\right)$ in state $j$ are exponential with parameter $j+1 / \theta^{2}, j=0,1, \ldots$ For $0 \leq t<1$ we have $Z_{t}=M_{-\ln (1-t)}$, so $-\log \left(1-\Gamma_{k}\right)=\sum_{j=0}^{k} \tau_{j}$. Therefore $\tau_{k}=\ln (1-$ $\left.\Gamma_{k-1}\right)-\ln \left(1-\Gamma_{k}\right)$ (here, we set $\left.\Gamma_{-1}=0\right)$. Since the Jacobian of the transformation $s_{j} \mapsto \ln \left(1-s_{j-1}\right)-\ln \left(1-s_{j}\right), j=0, \ldots, k$ is $J\left(s_{0}, \ldots, s_{k}\right)=\prod_{j=0}^{k}\left(1-s_{j}\right)^{-1}$, and $\tau_{0}, \tau_{1}, \ldots, \tau_{k}$ are independent, the joint density of $\left(\Gamma_{0}, \Gamma_{1}, \ldots, \Gamma_{k}\right)$ is

$$
J\left(s_{0}, \ldots, s_{k}\right) \prod_{j=0}^{k}\left(\left(j+1 / \theta^{2}\right) \exp \left(-\left(j+1 / \theta^{2}\right) \ln \frac{1-s_{j-1}}{1-s_{j}}\right)\right)
$$

which simplifies to (11).

Alternatively, we can use the fact that

$$
\begin{gathered}
R=P\left(s_{0}<\Gamma_{0}<s_{1}<\Gamma_{1}<s_{2}<\ldots<s_{k-1}<\Gamma_{k-1}<s_{k}<\Gamma_{k}\right) \\
=P\left(Z_{s_{0}}=0, Z_{s_{1}}=1, \ldots, Z_{s_{k}}=k\right) .
\end{gathered}
$$

The formula

$$
f_{\left(\Gamma_{0}, \Gamma_{1}, \ldots, \Gamma_{k}\right)}\left(s_{0}, s_{1}, \ldots, s_{k}\right)=(-1)^{k} \frac{\partial^{k+1} R}{\partial s_{k} \partial s_{k-1} \ldots \partial s_{1} \partial s_{0}}
$$

yields (11) after a calculation.

Proposition 4.4 (Distribution of downward jumps). Consider

$$
\Delta_{i}=\inf \left\{t>1: Z_{t}=i\right\}, \quad i=0,1, \ldots,
$$

i.e. $\Delta_{i}$ is the time of entrance of the process $\left(X_{t}\right)$ onto the line $y=\theta(t-1) i-1 / \theta$, $t>1$, for $i=0,1, \ldots$. Then the joint density of the random vector $\left(\Delta_{0}, \Delta_{1} \ldots, \Delta_{k}\right)$ is

$$
f_{\left(\Delta_{0}, \Delta_{1} \ldots, \Delta_{k}\right)}\left(t_{k}, \ldots, t_{1}, t_{0}\right)=\frac{\Gamma\left(\frac{1}{\theta^{2}}+k+1\right)\left(t_{k}-1\right)^{\frac{1}{\theta^{2}}+k-1}}{\Gamma\left(\frac{1}{\theta^{2}}\right) t_{k}^{\frac{1}{\theta^{2}}+k+1}\left(t_{0}-1\right)^{2}\left(t_{1}-1\right)^{2} \ldots\left(t_{k-1}-1\right)^{2}}
$$

for $1<t_{k}<\ldots<t_{1}<t_{0}$ (and 0 otherwise). 
Proof. From time-invertibility of the process, $\left(\Delta_{0}, \Delta_{1} \ldots, \Delta_{k}\right)$ has the same distribution as $\left(1 / \Gamma_{0}, 1 / \Gamma_{1}, \ldots, 1 / \Gamma_{k}\right)$. Thus

$$
f_{\left(\Delta_{0}, \Delta_{1} \ldots, \Delta_{k}\right)}\left(t_{k}, \ldots, t_{1}, t_{0}\right)=\frac{1}{t_{0}^{2} t_{1}^{2} \ldots t_{k}^{2}} f_{\left(\Gamma_{0}, \Gamma_{1}, \ldots, \Gamma_{k}\right)}\left(1 / t_{0}, 1 / t_{1}, \ldots, 1 / t_{k}\right),
$$

which simplifies to the expression above.

Proposition 4.5 (Time to reach lower boundary). The time a bi-Poisson process $\left(X_{t}\right)$ with parameters $\eta=\theta>0, q=1$ reaches the horizontal line $-1 / \theta$ on which it stays forever is finite but has infinite expectation.

Proof. The distribution of $\Delta_{0}=\inf \left\{t>1: X_{t}=-1 / \theta\right\}$ is a special case of the distribution of jumps, but it is just as easy to derive it independently. Since $Z_{t}$ is negative binomial, for $t>1$ we have $\operatorname{Pr}\left(\Delta_{0}>t\right)=1-\operatorname{Pr}\left(Z_{t}=0\right)=1-(1-1 / t)^{1 / \theta^{2}}$.

From the inequalities $(1-x)^{p} \leq 1-p x$ when $0<p<1, x \geq 0$ and $(1-x)^{p} \leq$ $(1-x)$ when $p>1,0 \leq x \leq 1$ we get

$$
\mathbb{E}\left(\Delta_{0}\right)=\int_{1}^{\infty}\left(1-\left(1-\frac{1}{t}\right)^{1 / \theta^{2}}\right) d t \geq \min \left\{1,1 / \theta^{2}\right\} \int_{1}^{\infty} \frac{d t}{t}=\infty .
$$

Proposition 4.6 (Poisson limit). For $\eta \theta>0$ let $\left(X_{t}^{(\eta, \theta)}\right)$ be the bi-Poisson process with parameters $(\eta, \theta)$, and let $\left(N_{t}\right)$ be the Poisson process with parameter $\lambda=1$. As $\eta \rightarrow 0$ the process $\left(\frac{1}{\theta} X_{t \theta^{2}}^{(\eta, \theta)}\right)$ converges in $D[0, \infty)$ to the Poisson-type process $\left(N_{t}-t\right)_{t \geq 0}$.

Proof. Calculating the conditional variances one can check that $\left(\frac{1}{\theta} X_{t \theta^{2}}^{(\eta, \theta)}\right)$ is a biPoisson process with parameters $(\eta \theta, 1)$

Consider now the bi-Poisson process $\left(X_{t}\right)=\left(X_{t}^{(\varepsilon)}\right)$ with parameters $(\varepsilon, \varepsilon)$ for $\varepsilon=\sqrt{\eta \theta}$. Then by the previous argument, process $Y^{\varepsilon}=\left(\frac{1}{\varepsilon} X_{t \varepsilon^{2}}^{(\varepsilon)}\right)_{t \geq 0}$ has the same distribution as process $\left(\frac{1}{\theta} X_{t \theta^{2}}^{(\eta, \theta)}\right)$. Therefore it suffices to show that as $\varepsilon \rightarrow 0$, the process $Y^{\varepsilon}$ converges in $D[0, \infty)$ to the Poisson-type process $\left(N_{t}-t\right)_{t>0}$.

We first verify the convergence of finite-dimensional distributions. For $0 \leq t<$ $1 / \varepsilon^{2}$, the appropriate version of (4) is

$$
\frac{1}{\varepsilon} X_{t \varepsilon^{2}}^{(\varepsilon)}=\left(1-t \varepsilon^{2}\right) Z_{t \varepsilon^{2}}^{(\varepsilon)}-t,
$$

where $Z_{t \varepsilon^{2}}^{(\varepsilon)}$ is a (non-homogeneous) pure birth process on $0 \leq t<1 / \varepsilon^{2}$. We will verify that the finite dimensional distributions of $\left(Z_{t \varepsilon^{2}}^{(\varepsilon)}\right)$ converge to the finite dimensional distributions of $\left(N_{t}\right)$.

Fix arbitrary $0=t_{0}<t_{1}<t_{2}<\cdots<t_{n}$ and $u_{1}, u_{2}, \ldots, u_{n} \leq 0$. It suffices to show that

$$
\lim _{\varepsilon \rightarrow 0} \mathbb{E}\left(\exp \left(\sum_{j=1}^{n} u_{j} Z_{t_{j} \varepsilon^{2}}^{(\varepsilon)}\right)\right)=\prod_{j=1}^{n} \exp \left(-\left(t_{j}-t_{j-1}\right)\left(e^{\sum_{i=j}^{n} u_{i}}-1\right)\right)
$$

We rely on the following observation, which can be regarded as special case of Slutsky's theorem: if $u_{\alpha} \rightarrow u$ and $\left(W_{1}^{(\alpha)}, W_{2}^{(\alpha)}, \ldots, W_{n}^{(\alpha)}\right)$ converges weakly to a 
random vector $\left(W_{1}, W_{2}, \ldots, W_{n}\right)$ as $\alpha \rightarrow 0$ and appropriate exponential moments exist, then

$$
\lim _{\alpha \rightarrow 0} \mathbb{E}\left(\exp \left(\sum_{j=1}^{n} u_{j} W_{j}^{(\alpha)}+u_{\alpha} W_{n}^{(\alpha)}\right)\right)=\mathbb{E}\left(\exp \left(\sum_{j=1}^{n} u_{j} W_{j}+u W_{n}\right)\right) .
$$

From (5) we have

$$
\mathbb{E}\left(\exp \left(\sum_{j=1}^{n} u_{j} Z_{t_{j} \varepsilon^{2}}^{(\varepsilon)}\right)\right)=A_{\varepsilon} \mathbb{E}\left(\exp \left(\sum_{j=1}^{n-1} u_{j} Z_{t_{j} \varepsilon^{2}}^{(\varepsilon)}+\left(u_{n}+u_{\varepsilon}\right) Z_{t_{n-1} \varepsilon^{2}}^{(\varepsilon)}\right)\right)
$$

where

and

$$
e^{u_{\varepsilon}}=\frac{1-\varepsilon^{2} t_{n}}{1-\varepsilon^{2} t_{n-1}-\varepsilon^{2}\left(t_{n}-t_{n-1}\right) e^{u_{n}}} \rightarrow 1
$$

$$
A_{\varepsilon}=e^{u_{\varepsilon} / \varepsilon^{2}} \rightarrow e^{-\left(t_{n}-t_{n-1}\right)\left(e^{u_{n}}-1\right)} .
$$

This proves (12) for $n=1$, and shows that (12) holding for $n-1$ implies (12) for $n$, ending the proof by induction.

Therefore, the increments of $\left(Z_{t \varepsilon^{2}}^{(\varepsilon)}\right)$ are asymptotically independent Poissondistributed with parameter $\lambda=t-s$, and the finite-dimensional distributions of $\left(Z_{t \varepsilon^{2}}^{(\varepsilon)}\right)$ converge to the corresponding distributions of $\left(N_{t}\right)$.

Tightness of $Y^{\varepsilon}$ in $D[0, \infty)$ follows from [7. Theorem 2.1 and Remark 4.2]. Indeed, their condition $\left(\mathrm{i}^{\infty}\right)$ holds as $Y^{\varepsilon}$ is a martingale and $E\left(Y^{\varepsilon}(t)\right)^{2}=t$. Their condition (ii ${ }^{\infty}$ ) holds as for fixed $a_{1} \leq s \leq a_{2}$ and all $\varepsilon$ small enough from (5) we get

$$
\begin{gathered}
\operatorname{Pr}\left(\text { at least two } Y^{\varepsilon} \text { jumps in }[s, s+\delta)\right) \\
=\operatorname{Pr}\left(X^{(\varepsilon)} \text { has at least two jumps in }\left[s \varepsilon^{2},(s+\delta) \varepsilon^{2}\right)\right) \\
\leq \operatorname{Pr}\left(Z_{(s+\delta) \varepsilon^{2}}^{(\varepsilon)}-Z_{s \varepsilon^{2}}^{(\varepsilon)} \geq 2\right) \leq \delta^{2} C\left(a_{1}, a_{2}\right) E\left(1+\varepsilon^{2} Z_{a_{2}}^{(\varepsilon)}\right)^{2} .
\end{gathered}
$$

Thus

$\delta^{-1} \limsup _{\varepsilon \rightarrow 0} \sup _{a_{1} \leq s \leq a_{2}} \operatorname{Pr}\left(\right.$ at least two $Y^{\varepsilon}$ jumps in $\left.[s, s+\delta)\right) \leq \delta C\left(a_{1}, a_{2}\right) \rightarrow 0$ as $\delta \rightarrow 0$.

Finally, their condition (iii) holds as for fixed $\eta>0$ and all $a>0$ small enough

$$
\limsup _{\varepsilon \rightarrow 0} \operatorname{Pr}\left(\sup _{s \leq a}\left|Y^{\varepsilon}(s)\right|>\eta\right) \leq \limsup _{\varepsilon \rightarrow 0} \operatorname{Pr}\left(Z_{a}^{(\varepsilon)} \geq 1\right)=a e^{-a} \rightarrow 0 \text { as } a \rightarrow 0 .
$$

Proposition 4.7 (Brownian limit). Let $\left(X_{t}\right)_{t \geq 0}=\left(X_{t}^{(\theta)}\right)_{t \geq 0}$ be the bi-Poisson process with parameters $\eta=\theta, q=1$. Then as $\theta \rightarrow 0$ the process $\left(X_{t}^{(\theta)}\right)_{t \geq 0}$ converges in $D[0, \infty)$ to the standard Brownian motion $\left(B_{t}\right)_{t \geq 0}$.

Proof. The convergence of finite dimensional distribution follows from uniqueness of the quadratic harnesses (see 12 for the special case we need here) and the fact that the limiting process must satisfy (3) by the uniform integrability of $\left\{\left(X_{t}^{(\theta)}\right)^{2}\right.$ : $\theta \leq 1\}$; in fact, $\mathbb{E}\left(X_{t}^{4}\right)=2 t^{2}+\left(t+3 t^{2}+t^{3}\right) \theta^{2}$.

Tightness in $D[0, \infty)$ now follows from [1] Proposition 1.2], as $\left(X_{t}^{(\theta)}: 0 \leq t<\infty\right)$ is a martingale for each $\theta$, and the limiting process is continuous. 
Here we give a simple direct argument for the convergence of finite dimensional distributions. It is enough to prove that for all $0=t_{0}<t_{1}<t_{2}<\cdots<t_{n}$ and all $u_{1}, u_{2}, \ldots, u_{n}$ close enough to zero

$$
\lim _{\theta \rightarrow 0} \mathbb{E}\left(\exp \left(\sum_{j=1}^{n} u_{j} X_{t_{j}}^{(\theta)}\right)\right)=\exp \frac{1}{2}\left(\sum_{k=1}^{n}\left(t_{k}-t_{k-1}\right)\left(\sum_{j=k}^{n} u_{j}\right)^{2}\right)
$$

We proceed by induction, suppressing $\theta$ in $X_{t}^{(\theta)}$ to shorten the expressions. Without loss of generality we may assume $t_{1}<1$ (set $u_{1}=0$, if necessary). To verify (14) for $n=1$ and $t_{1}<1$ we use (4) and (5) with $s=0$, which gives

$$
\mathbb{E}\left(e^{u_{1} X_{t_{1}}}\right)=\left(\frac{1-t_{1}}{e^{u_{1} t_{1} \theta}-t_{1} e^{u_{1} \theta}}\right)^{1 / \theta^{2}} \rightarrow \exp \left(\frac{1}{2} u_{1}^{2} t_{1}\right) .
$$

Suppose (14) holds for $0=t_{0}<t_{1}<t_{2}<\cdots<t_{n-1}<1$ and let $t_{n} \in\left(t_{n-1}, 1\right)$. Then again using (4) and (5) we get

$$
\mathbb{E}\left(\exp \left(\sum_{j=1}^{n} u_{j} X_{t_{j}}\right)\right)=A_{\theta} \mathbb{E}\left(\exp \left(\sum_{j=1}^{n-1} u_{j} X_{t_{j}}+u_{\theta} X_{t_{n-1}}\right)\right),
$$

where

$$
\begin{gathered}
A_{\theta}=\left(\frac{1-t_{n}}{1-t_{n-1}-\left(t_{n}-t_{n-1}\right) e^{\theta u_{n}\left(1-t_{n}\right)}}\right)^{1 /\left(\theta^{2}\left(1-t_{n-1}\right)\right)} \exp \left(\frac{-u_{n}\left(t_{n}-t_{n-1}\right)}{\theta\left(1-t_{n-1}\right)}\right), \\
e^{u_{\theta}}=\left(\frac{1-t_{n}}{1-t_{n-1}-\left(t_{n}-t_{n-1}\right) e^{\theta u_{n}\left(1-t_{n}\right)}}\right)^{1 /\left(\theta\left(1-t_{n-1}\right)\right)} \exp \left(u_{n} \frac{1-t_{n}}{1-t_{n-1}}\right)
\end{gathered}
$$

Since

$$
A_{\theta}=\left(\frac{1-t_{n}}{\left(1-t_{n-1}\right) e^{\theta u_{n}\left(t_{n}-t_{n-1}\right)}-\left(t_{n}-t_{n-1}\right) e^{\theta u_{n}\left(1-t_{n-1}\right)}}\right)^{1 /\left(\theta^{2}\left(1-t_{n-1}\right)\right)},
$$

by Taylor expansion $\lim _{\theta \rightarrow 0} A_{\theta}=e^{\frac{1}{2} u_{n}^{2}\left(t_{n}-t_{n-1}\right)}$. Similarly $\lim _{\theta \rightarrow 0} u_{\theta}=u_{n}$, which using (13) and induction assumption proves (14).

Now we know that (14) holds for all $n \geq 1$ and $0=t_{0}<t_{1}<t_{2}<\cdots<t_{n-1}<1$, and we take $t_{n}=1$. Using (4) and (6) we see that (15) holds with

$$
e^{u_{\theta}}=\left(\frac{1}{1-\left(1-t_{n-1}\right) u_{n} \theta}\right)^{1 /\left(\theta\left(1-t_{n-1}\right)\right)} \rightarrow e^{u_{n}}
$$

and

$$
A_{\theta}=e^{\left(u_{\theta}-u_{n}\right) / \theta} \rightarrow e^{\frac{1}{2} u_{n}^{2}\left(1-t_{n-1}\right)} .
$$

By (13) this proves (14).

Now we know that (14) holds for all $0=t_{0}<t_{1}<t_{2}<\cdots<t_{n-2}<t_{n-1}=1$, $n \geq 1$ and we take $t_{n}>1$. By (4) and (7) we get (15) with

$$
e^{u_{\theta}}=\exp \left(\frac{e^{\theta u_{n}\left(t_{n}-1\right)}-1}{\theta\left(t_{n}-1\right)}\right) \rightarrow e^{u_{n}}
$$

and

$$
A_{\theta}=e^{\left(u_{\theta}-u_{n}\right) / \theta} \rightarrow e^{\frac{1}{2} u_{n}^{2}\left(t_{n}-1\right)} .
$$

Again using (13) we get (14). 
Finally, we assume that (14) holds for all $0=t_{0}<t_{1}<t_{2}<\cdots<t_{n-1}, n \geq 1$ with $t_{n-1}>1$ and we take $t_{n}>t_{n-1}$.

Using (4) and (6) we see that (15) holds with

$$
e^{u_{\theta}}=\left(\frac{t_{n}-t_{n-1}+\left(t_{n-1}-1\right) e^{\theta u_{n} t_{n}-1}}{t_{n}-1}\right)^{1 /\left(\theta\left(t_{n-1}-1\right)\right)} \rightarrow e^{u_{n}}
$$

and

$$
A_{\theta}=e^{\left(u_{\theta}-u_{n}\right) / \theta} \rightarrow e^{\frac{1}{2} u_{n}^{2}\left(t_{n}-t_{n-1}\right)} .
$$

By (13) this proves (14).

Acknowledgement. We would like to thank Wojciech Matysiak for comments and discussions and Sergiusz Wesołowski for assistance with simulations of the biPoisson process in Fig. 11

\section{REFERENCES}

[1] David Aldous. Stopping times and tightness II. The Annals of Probability, 17(2):586-595, 1989.

[2] Włodzimierz Bryc, Wojciech Matysiak, and Jacek Wesołowski. Quadratic harnesses, qcommutations, and orthogonal martingale polynomials. arxiv.org/abs/math.PR/0504194, 2005.

[3] Włodzimierz Bryc, Wojciech Matysiak, and Jacek Wesołowski. Quadratic harnesses: the biPoisson processes (tentative title). In preparation, 2005.

[4] Włodzimierz Bryc and Jacek Wesołowski. Bi-Poisson process. Submitted. arxiv.org/abs/math.PR/0404241, 2004.

[5] Włodzimierz Bryc and Jacek Wesołowski. Conditional moments of $q$-Meixner processes. Probability Theory Related Fields, 131:415-441, 2005. arxiv.org/abs/math.PR/0403016.

[6] L. Gallardo and M. Yor. Some new examples of Markov processes which enjoy the timeinversion property. Probability Theory Related Fields, 132:150-162, 2005.

[7] Allan Gut and Svante Janson. Tightness and weak convergence for jump processes. Statist. Probab. Lett., 52(1):101-107, 2001.

[8] Stephan Lawi. A characterization of Markov processes enjoying the time-inversion property, 2005. arXiv:math.PR/0506013.

[9] R. Mansuy and M. Yor. Harnesses, Lévy bridges and Monsieur Jourdain. Stochastic Processes and Their Applications, 115:329-338, February 2005.

[10] Emanuel Parzen. Stochastic processes. Holden-Day Series in Probability and Statistics. Holden-Day Inc., San Francisco, Calif., 1962.

[11] Shinzo Watanabe. On time inversion of one-dimensional diffusion processes. Z. Wahrscheinlichkeitstheorie und Verw. Gebiete, 31:115-124, 1974/75.

[12] Jacek Wesołowski. Stochastic processes with linear conditional expectation and quadratic conditional variance. Probab. Math. Statist., 14:33-44, 1993.

Department of Mathematics, University of Cincinnati, PO Box 210025, Cincinnati, $\mathrm{OH}$ 45221-0025, USA

E-mail address: Wlodzimierz.Bryc@UC.edu

Faculty of Mathematics and Information Science, Warsaw University of Technology, Pl. Politechniki 1, 00-661 Warszawa, Poland

E-mail address: wesolo@alpha.mini.pw.edu.pl 